\title{
Respuesta de crecimiento y floración de Tagetes erecta "San Diego" mediante la agregación de fertilización carbónica atmosférica bajo condiciones controladas Estación Experimental "El Limón"
}

\author{
Josué Urrutia ${ }^{1}$
}

\section{RESUMEN}

El establecimiento plantaciones ornamentales, cultivos agrícolas y plantaciones forestales pueden estar afectados por los cambios en el clima que conlleva a un estrés de los organismos y bajar los índices de producción. Este trabajo evalúa los beneficios del gas conocido como dióxido de carbono en su uso como fertilizante atmosférico y conocer sus efectos sobre el cultivo de la planta San Diego (Tagentes erecta). Para el establecimiento del ensayo se utilizaron las instalaciones de la Estación Experimental "El Limón” propiedad de la FAREM-Estelí/UNAN-Managua. Al evaluar los efectos de la fertilidad del $\mathrm{CO} 2$, se realizó dentro de microtuneles donde se hicieron las restricciones de este gas, se midió: el crecimiento de la planta en altura y diámetro basal, materia seca, índice de robustez y calidad. Se tomó como referencia la Evaluación del Indice de calidad de Dickson (1960). Los resultados demostraron que no existe diferencia sobre la altura y diámetro basal de las plantas, las dosis que sobre pasan los 400ppm de $\mathrm{CO} 2$ aumentan la materia seca de este cultivo ornamental, y esta se incrementa entre un 6 y 7\%. Los índices de esbeltez y calidad indican que las plantas sometidas a 400ppm de $\mathrm{CO} 2$ son las que podrían sobrevivir mejor en condiciones de climas adversos al ser establecidas en campo.

Palabras claves: Tagetes erecta, Fertilización carbónica, Biomasa seca y calidad de plantas.

Recibido: 3 de marzo de 2016

Aceptado: 4 de abril de 2016

1 UNAN-Managua/FAREM-Estelí. Correo Electrónico: josuerod20@yahoo.com 


\title{
Response of the growth and flowering of Tagetes erecta "San Diego" by aggregating atmospheric carbon dioxide fertilization under controlled conditions at the Experimental Station "El Limón"
}

\begin{abstract}
The establishment: ornamental plantings, agricultural crops and forest plantations can be affected by climate changes that leads to a stress of organisms and lower production rates. This paper assesses the benefits of gas known as carbon dioxide used as atmospheric fertilizer use and know their effects on the cultivation of San Diego plant (Tagentes erecta). For the establishment of the research, the facilities of the Experimental Station "El Limón" managed by FAREM -Estelí / UNAN - Managua were used. In assessing the effects of CO2 fertility, Micro tunnels were used, where restrictions of this gas were made. The data measured was: the growth of plant (height and basal diameter), dry matter, robustness and quality index. It was taken as reference the Assessment Quality Index of Dickson (1960). The results showed no difference on height and basal diameter of the plants, the doses which surpass 400 ppm of CO2 increase the dry material of this ornamental crop, and this increases between 6 and 7\%. Slenderness rates and quality indicate that plants under $400 \mathrm{ppm} \mathrm{CO} 2$ are what could best survive in adverse conditions to be established in the field climates.
\end{abstract}

Keywords: Tagetes erecta, carbonic fertilization, dry biomass and plant quality. 


\section{INTRODUCCIÓN}

El cambio climático es un fenómeno importante porque está provocando estrés a los organismos (plantas y animales). Aunque no todas las plantas estarán afectadas en su estado fenológicos al mismo tiempo, ni en la misma intensidad. De acuerdo a Prado (2010) lo más importante que destaca son: el estrés abiótico, biótico, antropogénico, y la combinación del estrés que se presenta el mismo espacio y al mismo tiempo, lo que puede causar efectos sinérgicos sobre la vegetación y en los cultivos.

El potencial de cambio climático atribuible al cambio global, puede aumentar la temperatura local y general (IPCC, 2007). Estos pequeños cambios de temperatura pueden tener gran influencia en el equilibrio de carbono atmosférico y por tanto en la fotosíntesis.

Una característica importante en las plantas verdes es la asimilación de CO2 (Anton, et al, 2011). Pero también hay que reconocer que en la actualidad el $\mathrm{CO} 2$ es conocido, por su efecto, como gas de efecto invernadero, que contribuye al calentamiento de la atmosfera y al cambio climático. Sin embargo, más que los efectos negativos de este gas, es que se destaca como una de las dos materias primas, junto con el agua, a partir de las cuales las plantas fabrican, en la fotosíntesis, su propia materia, de la cual dependen la mayoría de los seres vivos, bien como alimento primario, o como alimento de su alimento. La vida se basa en el carbono y el $\mathrm{CO} 2$ es la fuente de carbono.

A nivel fisiológico, la mayor concentración de bióxido de carbono en la atmósfera, en los océanos ha tenido consecuencias importantes en los procesos de alimentación y crecimiento de muchas especies vegetales. Algunas especies de plantas, forestales, ornamentales y cultivos agrícolas, se han beneficiado debido a que han sido capaces de absorber e integrar en sus tejidos una mayor cantidad de este gas (INECC, 2012)
En ese contexto, es fácil comprender que la exposición de las plantas a ambientes con una elevada concentración de dióxido de carbono durante cortos períodos de tiempo, generalmente estimula el crecimiento y la fijación fotosintética de carbono (Anton, et al, 2011). Así, en estudios a corto plazo (inferiores a los 6 meses) en árboles expuestos a niveles elevados de $\mathrm{CO} 2$ y con diferentes disponibilidades de recursos, se ha descrito que la biomasa total incrementa de media un $38 \%$ en coníferas y en un $63 \%$ en caducifolios (Carnovky et al, 2011).

Igualmente, en estudios llevados a cabo a medio y/o a largo plazo se han descrito cambios en el tamaño y en las relaciones alométricas (Bazzaz et al., 1993), en la cantidad y proporción de biomasa radical (Anton, et al, 2011) y en la concentración de diferentes nutrientes en los tejidos, especialmente nitrógeno (Ramírez et al, 2015).

Por ello, la técnica del enriquecimiento carbónico, también denominada fertilización carbónica, consiste en aumentar el nivel ambiental de $\mathrm{CO} 2$ hasta aproximadamente doblar las cantidades actuales contenidas en la atmosfera. La mayoría de los estudios del efecto que tiene la aplicación de $\mathrm{CO} 2$, se ha enfocado en los aspectos de distribución de biomasa, obtención de metabolitos secundarios con interés farmacológicos.

Teniendo en cuenta que entre el 45-50\% del peso seco de la planta es carbono, la agricultura se puede convertir en un mecanismo efectivo para mitigar el incremento del $\mathrm{CO} 2$ atmosférico. La fertilización con $\mathrm{CO} 2$ permite el mayor crecimiento, producción de flores y frutos, además del aumento del peso específico se ve favorecida con el aumento con la mayor dosificación las que pueden ser hasta de 750 ppm.

Este estudio permitió determinar la respuesta de adaptación, crecimiento y floración de Tagetes erecta 
San Diego, mediante la agregación de fertilización carbónica atmosférica en especies ornamentales y alimenticias, bajo condiciones controladas Estación Experimental "El Limón", durante el primer semestre del 2015.

El principal problema es la falta de reproducción de especies ornamentales necesarias para la recuperación de especies y el conocimiento del efecto que puede tener el cambio climático en las diferentes especies ornamentales, ya sea que estén en vías desaparecer o no y la satisfacción del mercado local (viveros, floristería y autoconsumo).

El objetivo de esta investigación ha sido evaluar la respuesta de adaptación, crecimiento y floración de Tagetes erecta (San Diego), mediante la agregación de fertilización carbónica atmosférica en especies ornamentales, bajo condiciones controladas Estación Experimental "El Limón". Se evaluaron los efectos en formas de gas, en el crecimiento y el efecto de una atmósfera enriquecida con dióxido de carbono en la calidad de plantas, flores y frutos de Tagetes erecta bajo condiciones controladas.

\section{MATERIAL Y MÉTODO}

Para la evaluación de la efectividad de la fertilización carbónica y lumínica se establecieron seis cajas plásticas a cielo abierto, con dimensiones de $1 \mathrm{x}$ 1.5 x 1.5 metros o 4.5 metros cúbicos. Se hicieron aplicaciones con prolongaciones de 2 minutos por cada uno de los pequeños invernaderos, donde se establecieron plantas ornamentales como San Diego. A cada planta se les hizo distintas aplicaciones de dióxido de carbono para reconocer que dosificación y tiempo tiene efecto sobre el crecimiento y producción de flores y frutos. Ver imagen 1.

\section{Imagen No 1. Diseño, estructura para realizar la fertilización carbónica.}

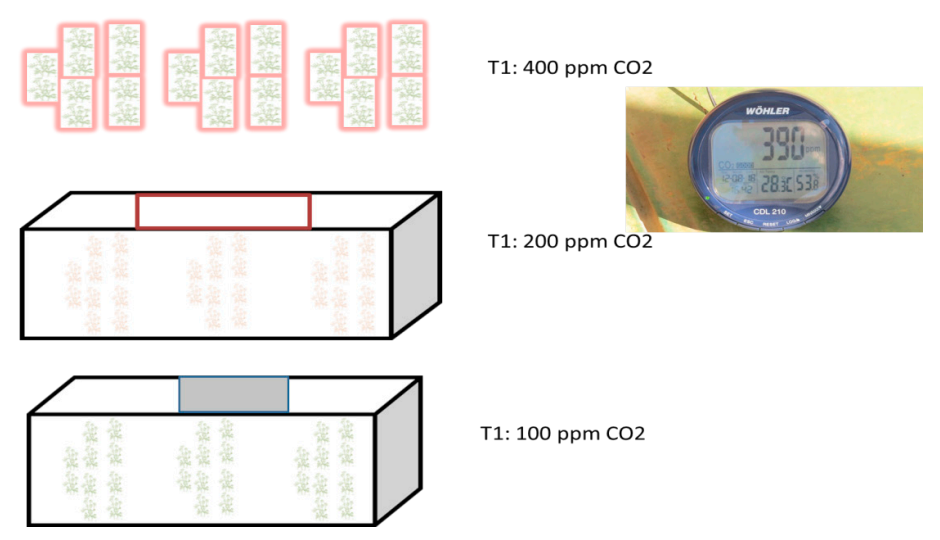

Para medir la producción de dióxido de carbono se utilizaron gases provenientes de empresas que se dedican a la venta y comercialización de los mismos. En la producción artesanal de $\mathrm{CO} 2$, se elaboraron reactores hechos a bases de botellas plásticas trasparentes de 2.5 litros, con diámetros internos de $14 \mathrm{~cm}$ y alturas de $35 \mathrm{~cm}$, con un volumen de cultivo de 2 litros. Los reactores inyectaron el gas, a lo interno de cada micro túnel, por medio de una manguerilla, mediante un flujo continuo, que duró una semana y aplicándolo solo en las horas de mayor luminosidad.

Cada uno de los tratamientos se realizó por triplicado por un periodo de 12 horas luz y 12 horas de oscuridad. Todos los experimentos tuvieron un tiempo de cultivo de 4 semanas.

Para conocer el efecto de la fertilización carbónica sobre las plantas de Tagetes erecta, se efectuaron al menos tres dosificaciones de dióxido de carbonos en forma de gas. Los tratamientos fueron: un testigo con el dióxido de carbono atmosférico, con una dosificación de 400 ppm; un segundo tratamiento con restricciones de dióxido de carbono bajando la cantidad a 200 ppm; y un tercer tratamiento con restricciones de $100 \mathrm{ppm}$. Estas dosificaciones y mediciones se realizaron de las 10:00 de la mañana a las 3:00 de la tarde. Se hizo la medición a las doce de medio día, por ser la hora de mayor demanda de por parte y es la hora que te marca 
la caída de gas en cuestión. Las mediciones de dióxido de carbono se realizaron utilizando un sensor de este gas marca Wöhller CDL 210.

En la medición de la calidad de la planta se consideraron criterios morfológicos y fisiológicos. La información que se registró por cada uno de los tratamientos fueron: altura $(\mathrm{cm})$, diámetro al cuello de la raíz $(\mathrm{mm}), \mathrm{y}$ Biomasa ( $\mathrm{g}$ ) en húmedo y en seco de la parte aérea. Los primeros tratamientos son los más utilizados por la facilidad para ser evaluada la raíz. Las muestras se deshidrataron a $70^{\circ} \mathrm{C}$, durante 72 horas. También se calculó la relación biomasa seca aérea/biomasa y seca de la raíz (BSA/BSR). La relación altura $(\mathrm{cm}) /$ diámetro $(\mathrm{mm})$, conocida como índice de robustez (IR), y el índice de Calidad de Dickson (ICD) reúne varios atributos morfológicos (altura, diámetro, peso seco y peso fresco) en un valor (Dickson et al 1960; Prieto et al., 2003) y se calcula de la siguiente formula:

$\mathrm{IC}=$ peso seco total $(\mathrm{g}) /$ altura $(\mathrm{cm}) /$ diámetro $(\mathrm{mm})+$ Peso seco de la parte aérea $(\mathrm{g}) /$ peso seco de la raíz (g).

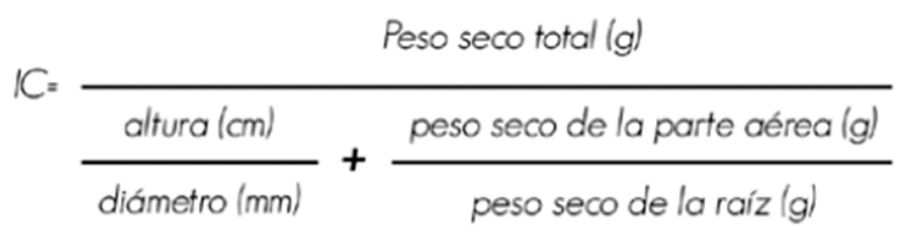

Entre esos criterios destacan la altura del tallo, el diámetro al cuello y la producción de biomasa, lo que a su vez permite estimar el índice de robustez (altura/ diámetro) y la relación biomasa parte aérea/raíz. Para las especies del género Pinus de clima templado frío, en general, se consideraron los indicadores: altura2: 13 a $25 \mathrm{~cm}$, diámetro al cuello: $\geq 4.0 \mathrm{~mm}$, índice de robustez: $<6$ y relación biomasa parte aérea/raíz: 1.5 a 2.5. Además, la planta debe ser joven (10 a 12 meses), el sistema radical debe tener puntos de crecimiento en abundancia, y estar sana y vigorosa.

\section{Plan de Análisis Estadístico}

De los datos que generados por ficha de recolección de datos sobre la fertilización carbónicas en los dos cultivares se realizó el análisis estadístico pertinente, según la naturaleza de c/u de las variables. Mediante el análisis de contingencia, (crosstab análisis, Pedroza 2014 para todas aquellas variables no paramétricas, a las que se les aplicó pruebas de Chi2 y/o de Phi, o de Kramer, o correlación de Spearman; a las variables ordinales.

Se realizó análisis gráficos del tipo: barras de manera uní y multivariadas, que describen en forma clara la interrelación de las variables.

Se aplicaron las estadísticas inferenciales específicas en las variables cuantitativas que cumplían con las condiciones de ser paramétricas y pertinentes al estudio, tales como: la prueba de Correlación de Pearson, el ANOVA univariado y el MANOVA,

\section{RESULTADOS Y DISCUCIÓN}

La aplicación o enriquecimiento con dióxido de carbono, llamada igualmente fertilización carbónica, utilizada en su mayoría a lo interno de los invernaderos en cultivos ornamentales, hortícolas, plantaciones forestales en desarrollo y otros tiene como propósito elevar los niveles de este gas a ser doblados o triplicados tomando en cuenta las concentraciones actuales que según los expertos son de 400 ppm. La utilización del $\mathrm{CO} 2$ es una técnica ampliamente utilizada en la industria de conservas, producción de cervezas, control de incendios, pero también se puede utilizar como una técnica agronómica desde hace unos años.

\section{Evaluación del Crecimiento de las plántulas de San Diego (Tagetes erecta)}

El crecimiento de Tagetes erecta se midió tomando en cuenta las comparaciones entre los diámetros y alturas 
por cada uno de los tratamientos no se logró percibir diferencias estadísticamente diferentes, esto puede deberse a que las variables consideradas no representan la calidad, ni productividad de las plántulas de San Diego, grafica 1 y 2.

Aunque no se encontró diferencia significativa, si se pudo observar un efecto positivo cuando las dosis son iguales a 100 ppm y 400 ppm, tanto para el diámetro como para las alturas de las plantas que fueron expuestas a estas dosis de $\mathrm{CO} 2$ dosis. Los resultados positivos de la adición más allá de las concentraciones atmosféricas se pueden notar en diferentes experimentos tanto con plantas forestales, frutales y ornamentales, las que han expresado un mayor crecimiento tanto en altura como en diámetro de las especies e individuos sometidos a estos tratamientos (Anton, et al, 2011).

Grafica 1 y 2: Efecto de la fertilización carbónica sobre el diámetro y altura de las plántulas de San Diego.

\section{Efecto de Dióxido de Carbono sobre el diametro de Tagetes erecta}

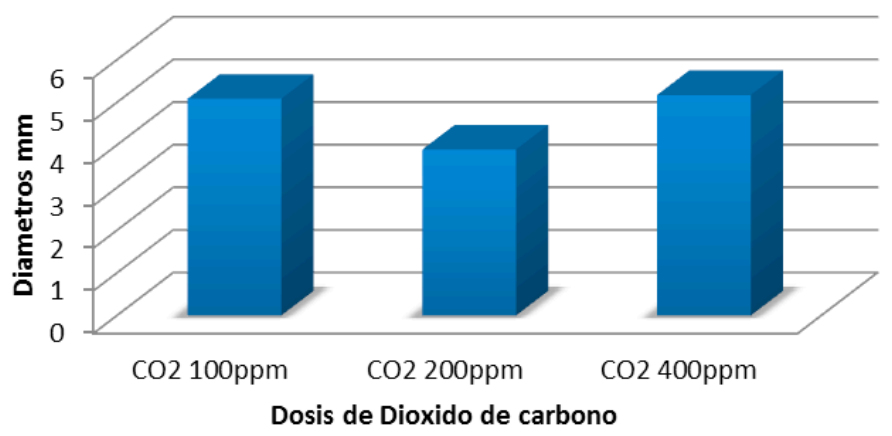

\section{Efecto de la fertilización carbónica sobre} la altura Tagetes erecta

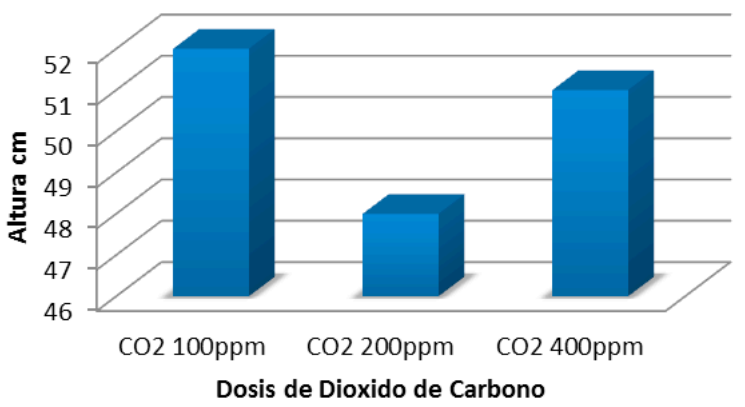

Altura

Fuente: Josué Tomás Urrutia Rodríguez. Fertilización carbónica en Tagetes erecta. Septiembre, 2015
Evaluación de la Biomasa fresca y seca de tallos y raíces en plántula de San Diego

Al realizar las comparaciones de los diferentes tratamientos en biomasa fresca de tallos y raíces se muestran que las plantas de Tagetes erecta que fue expuesta a mayores dosis de $\mathrm{CO} 2$, aumentan su contenido de biomasa seca, presentando una mayor cantidad de biomasa fresca las plántulas sometidas a condiciones ambientales con cantidades de dióxido de carbono que oscila entre los 392 y 400 ppm. Con una significancia en raíces de $\mathrm{P}=0.427$ y en tallos presentan la misma tendencia en cuanto a valores de significancia el cual es $\mathrm{P}=0.0162$. Grafica 3 .

Gráfica 3. Evaluación de biomasa fresca en raíces y tallos de Tagetes erecta

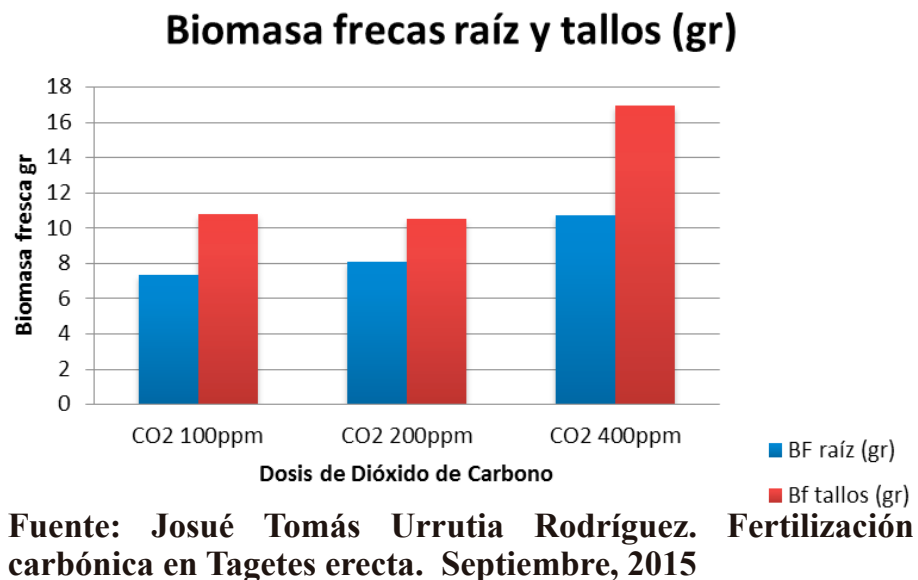

Evaluación de biomasa seca en raíces y tallos de Tagetes erecta.

Cuando se comparan los diferentes tratamientos en cuanto a biomasa seca de tallos y raíces se encontró que las plantas de Tagetes erecta que fueron expuestas a mayores dosis de $\mathrm{CO} 2$, aumentan su contenido de biomasa seca. 
Gráfica 4. Evaluación de materia seca de raíces y tallos en Tagetes erecta.

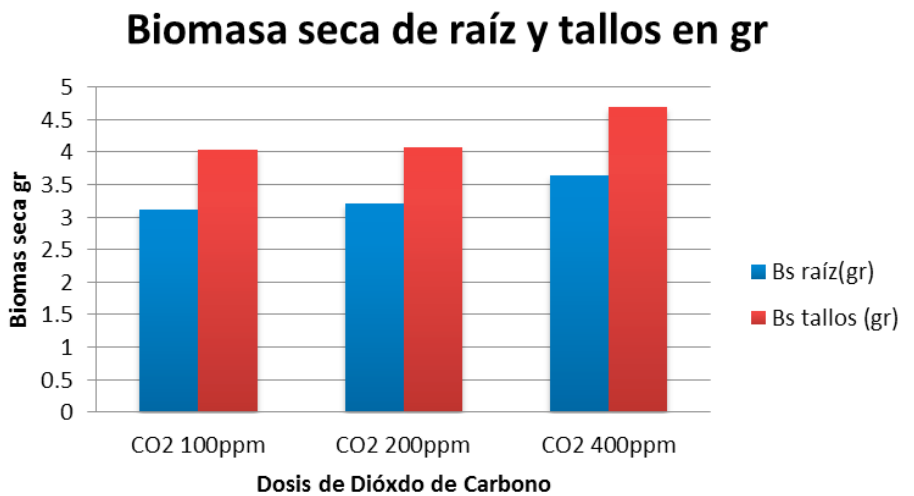

Fuente: Josué Tomás Urrutia Rodríguez. Fertilización carbónica en Tagetes erecta. Septiembre, 2015

$\mathrm{Al}$ establecer relaciones entre biomasa fresca y seca se observaron cambios significativos por el aumento tanto de biomasa seca en la parte aérea como de raíces en las plantas expuestas a mayores dosificaciones de dióxido de carbono, lo cual puede estar dado por lo afirmado por Sánchez-González (2013) que este tipo de fertilización puede ser positiva para el crecimiento de las plantas lo que se refleja en una mayor acumulación de materia seca (Bindi et al, 2001) lo cual puede estar estimulado por los altos niveles en las de concentración de $\mathrm{CO} 2$ aumentado de esta manera la absorción de elementos esenciales lo que conlleva una alta tasas de crecimiento resultante (Segura M. L. et al., 2009) y culmina en la mejora en el uso eficiente y baja concentración de los nutriente en los tejidos.

Al hacer comparaciones de los diferentes tratamientos de materia seca de tallos y raíces se observó que las plantas de Tagetes erecta expuestas a mayores dosis de $\mathrm{CO} 2$, aumentan su contenido de biomasa seca entre un 6 y 7\%. Gráfica No.5.
Grafica 5. Evaluación de materia seca de raíces y tallos de Tagetes erecta.

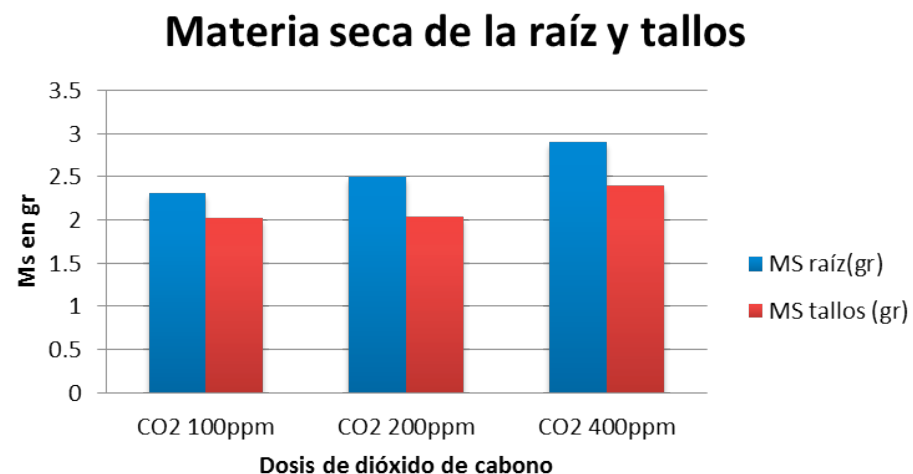

Fuente: Josué Tosis de dióxido de cabono carbónica en Tagetes erecta. Septiembre, 2015

\section{Evaluación de la fertilización carbónica en plantas de Tagetes erecta en base al Índice de Robustez:}

Las plantas de San Diego sometidas 400ppm de CO2 son las que presentan el menor índice de resistencia, por ser un índice inversamente proporcional, tienen la mejor cualidad para enfrentarse a condiciones ambientales adversas, y se pueden adaptar a condiciones extremas. Mientras que presentan un medio y bajo índice de robustez las plantas que se sometieron a 100 y 200 ppm de dióxido de carbono.

Grafica 6. Evaluación de índice de robustez en plantas de Tagetes erecta Indice Robustez en plantas de San Diego

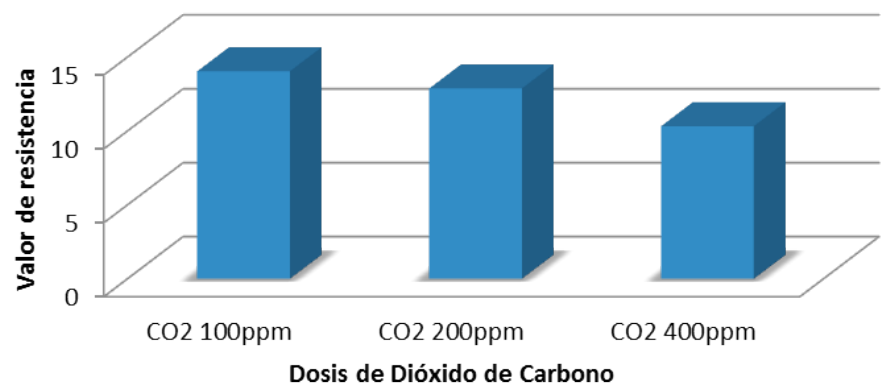

Fuente: Josué Tomás Urrutia Rodríguez. Fertilización carbónica en Tagetes erecta. Septiembre, 2015

\section{Evaluación del Índice de Calidad de Dickson en plantas de Tagetes erecta}

Cuando se integra los valores de biomasa total, y el índice de esbeltez da como resultado el índice de calidad 
de plantas (Dickson et al 1960). Mostrando las plantas de San Diego sometidas 400ppm de CO2 y que tiene un índice de calidad de 5.4 y que según (Thompson, 1985) los valores más altos pertenecen a las plantas de mayor calidad con respecto a las plantas sometidas a los demás tratamientos. Con lo cual podemos predecir la superioridad en calidad de las plantas tratadas con dosis altas de CO2 (Mateo-Zánches et al., 2011), Pero que a la vez las fracciones aéreas y radicales están equilibradas (Oliet, 2000).

Grafico 6. Evaluación del índice de calidad de Dickson en plantas de Tagetes erecta Índice de calidad de Dickson

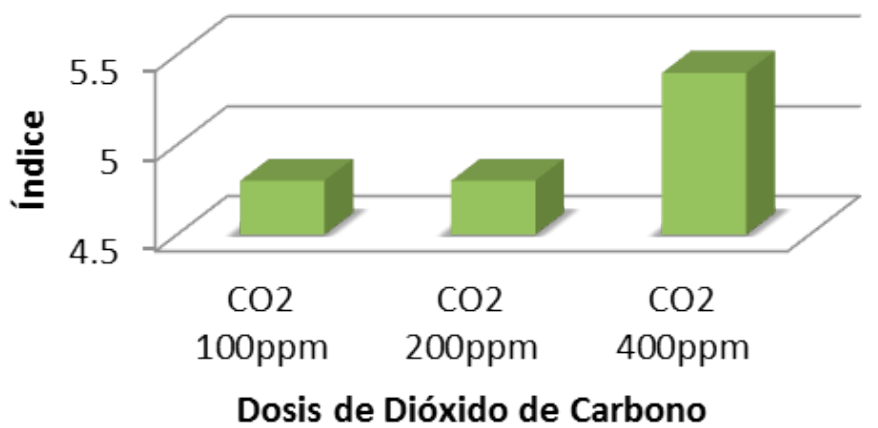

Fuente: Josué Tomás Urrutia Rodríguez. Fertilización carbónica en Tagetes erecta. Septiembre, 2015

\section{CONCLUSIONES}

La aplicación fertilizante a base de dióxido de carbono es una alternativa en la promoción de la agricultura orgánica y el reciclaje de este gas, porque promueve el crecimiento de la planta, aumenta la cantidad de biomasa de la planta y mejora los índices de robustez y calidad del cultivo.

El sometimiento de las plantas de Tagetes erecta a 400 ppm de dióxido de carbono aumenta significativamente la productividad de las plantas entre un 6-7\%.

\section{AGRADECIMIENTO}

Este estudio se llevó a cabo gracias al financiamiento del Programa de Naciones Unidas (PNUD)

\section{BIBLIOGRAFÍA}

Anton, et al. (2011). Manual del aplicador de aplicador de $\mathrm{CO} 2$ en hotalizas. www.recerca.cat, 36.

Bindi et al, M. (2001). Growth and quality of grape and wine in response to elevate $\mathrm{CO} 2$ concentrations. En Bindi, Free Air CO2E Enrichment (FACE) of grapevine (Vitis vinifera L.) (págs. 14. 145-155). Eur. J. Agron.

Carnovky et al. (2011). Inpact of carbono Dioxide and other Greenhause gases and forest ecosystem. $C A B$ international, 74.

INECC. (2012). Adaptación al Cambio Climático en México. Visión, elementos y criterios para toma de decisiones. México.

IPCC. (2007). Cambio climático 2007 Informe y síntesis. IPCC, 144.

Mateo-Zánches et al., J. J. (2011). Producción de (Cedrela odorata L.), en sustratos a base de aserrín crudo en sistemas tecnificados en Tecpan de Galeana, Guerrero, México. Ra Ximhai, 11.

Oliet, J. (2000). La calidad de la postura forestal en vivero. Escuela Tecnica Superior de Ingenieros de Montes de Cordoba. España, 93.

Prado et al. (2010). Las especies C4 y el estres ambiental. scis, 11.

Ramírez et al. (2015). Responses of fruit trees to global climate change. New York: Springerbrief in plant science.

Sánchez-González, L. (2013). Influencia del enriquesimiento carbónico y la salinidad sobre la producción de tomate cv.Deizia (híbrido RAF). Congreso Iberico de Agroingenieria y Ciencias Hortícolas, Madrid., (pág. 7). Madrid.

Segura M. L. et al. (2009). Influence of salinity and fertilization level on the distribution en tomato plants under a polyethylene greenhose in the Mediterranean area. Soil Sci. Plant, 40: 498-513.

Thompson, B. (1985). Seedling Morphological evaluación: What you can tell by looking. In: Evaluating seedling quality: Principles, procedures, and Predictive abienties of major test. M.L. Duryea Forest Res.Lab, 59-71. 